\section{Evaluation of ocular pulse amplitude and choroidal thickness in diabetic macular edema}

\begin{abstract}
Purpose The aim of this study is to evaluate the ocular pulse amplitude (OPA) and choroidal thickness (CT) measurements in patients with diabetic macular edema (DME) and healthy subjects.

Methods A total of 34 patients (12 male and 22 female) who had type 2 diabetes mellitus with DME and 34 sex-matched healthy subjects (13 male and 21 female) were included in this prospective study. The intraocular pressure (IOP) and OPA were measured with Dynamic contour tonometer (Pascal DCT, Switzerland). The subfoveal CT was measured using the Cirrus HD-OCT

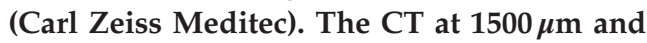
$3000 \mu \mathrm{m}$ nasal and temporal to the central fovea was also measured.

Results The mean IOP values were $18.4 \pm 3.5$ and $17.1 \pm 2.1 \mathrm{~mm} \mathrm{Hg}$ in DME patients and healthy controls, respectively $(P=0.091)$. The mean OPA values in patients with DME $(2.58 \pm 0.96)$ and controls $(3.52 \pm 1.03)$ were statistically different $(P<0.001)$. The mean subfoveal CT value was $273.5 \pm 30.2 \mu \mathrm{m}$ in the eyes with DME and $321.4 \pm 36.5 \mu \mathrm{m}$ in the control group $(P<0.001)$. In both groups, linear regression analysis showed no significant association between OPA and CT measurements.

The IOP showed a significantly positive correlation with OPA in both DME $(P=0.002$, $r=0.526)$ and controls $(P=0.004, r=0.483)$. Conclusions The current study suggests that both pulsatile choroidal blood flow and CT are decreased in patients with DME.

retinopathy worldwide. ${ }^{1}$ Although alterations in retinal vasculature resulting in compromise of the blood-retinal barrier have been demonstrated to have a critical role in the pathophysiology of the disease, changes in the choroidal vasculature may also have a contributing role.

Detailed visualization of the choroid is now possible with the spectral domain-optical coherence tomography (SD-OCT) imaging. Recent studies applying SD-OCT to evaluate choroidal thickness (CT) in eyes with DME have demonstrated altered and inconsistent CT measurements. ${ }^{2-7}$ One important explanation for these variable results is these studies include eyes that received treatment with intravitreal anti-vascular endothelial growth factor (antiVEGF) therapy, which has been shown to cause choroidal thinning. ${ }^{3}$ Recently, baseline subfoveal CT was shown to help predict which patients with DME will respond more favorably in the short term to intravitreal anti-VEGF pharmacotherapy. ${ }^{8}$

Ocular pulse amplitude (OPA) is the difference between diastolic and systolic intraocular pressure (IOP) and represents the magnitude of change in IOP with the ocular pulse. OPA, caused by cardiovascular pulsations and ocular blood flow, is an index of choroidal perfusion. ${ }^{9,10}$ Despite growing evidence demonstrating alterations to the choroidal vasculature in DME, the relationship between CT and OPA has not been studied. The aim of this study is to evaluate and compare the OPA and CT measurements in treatment-naive eyes with DME and healthy subjects.
\end{abstract}

Eye (2016) 30, 369-374; doi:10.1038/eye.2015.232; published online 13 November 2015

\section{Introduction}

Diabetic macular edema (DME) is the leading cause of blindness in patients with diabetic

\section{Materials and methods}

A total of 34 patients (12 male and 22 female) who had type 2 diabetes mellitus with DME and 34 sex-matched healthy subjects ( 13 male and 21 female) were included in this prospective study.

\author{
'Department of \\ Opthalmology, Medical \\ School, Turgut Özal \\ University, Ankara, Turkey \\ ${ }^{2}$ Erciş State Hospital, Eye \\ Clinic, Van, Turkey \\ ${ }^{3}$ Gölbaşı Hasvak State \\ Hospital, Eye Clinic, \\ Ankara, Turkey \\ Correspondence: \\ E Güler, Ophthalmology, \\ Ercis State Hospital, Eye \\ Clinic, Van Yolu Cad. No: \\ 57, Van 65400, Turkey \\ Tel: +90 43235196 61; \\ Fax: +90 3122213276 . \\ E-mail: guleremre83@ \\ hotmail.com
}

Received: 12 February 2015 Accepted in revised form: 8 September 2015 Published online: 13 November 2015 
We have recruited all the patients from our retina department, in University of Turgut Özal, from December 2013 to August 2014. DME was diagnosed by the examining physician as defined by the Early Treatment Diabetic Retinopathy Study. ${ }^{11}$ Center-involving DME with central macular thickness over $275 \mu \mathrm{m}$ on OCT was also required for diagnosis. ${ }^{12}$ Inclusion criteria were age over 18 years, a best-corrected visual acuity (BCVA) between 1.0 and $0.3(\log \mathrm{MAR})$ and without prior antiVEGF therapy. The exclusion criteria were a history of glaucoma, severe cataract, venous occlusions, epiretinal membrane visible by OCT, age-related macular degeneration, uveitis, history of cataract surgery (within the previous 6 months), YAG laser capsulotomy (within 2 months prior to the trial), previous vitrectomy, DME previously treated with recent panretinal or grid laser photocoagulation, and intravitreal or periocular corticosteroids (within 3 months prior to investigation). Only one eye from each subject was included for analysis. If both eyes qualified, the eye with worse BCVA was selected. All examinations were performed between 1300 to 1500 hours to avoid diurnal variations. ${ }^{13}$

Pertinent clinical data recorded included patient age, sex, hypertension, duration of diabetes mellitus, baseline and follow-up BCVA, complete biomicroscopic examination findings, spherical equivalent (SE) values of refractive errors and axial length (AL) measured by using optical biometer (AL scan). An informed consent was obtained from the subjects. This study was approved by the Ethics Committee and was conducted in accordance with the Declaration of Helsinki Principles.

The OPA and IOP measurements were done with the dynamic contour tonometer (Pascal DCT; Swiss Microtechnology AG, Port, Switzerland). This noninvasive contact device is attached to a slit-lamp biomicroscope and it has a 7-mm tip diameter and a $1.2-\mathrm{mm}$ pressure sensor diameter. OPA was calculated as the difference between the systolic and diastolic IOP. Three DCT measurements were performed to achieve a good-quality measurement (quality level 1-2) and the data from the better quality measurement were taken for analysis.

The CT measurements were performed by using the Cirrus HD-OCT (Carl Zeiss Meditec Ophthalmic Systems Inc., Dublin, CA, USA), which uses light at 840-nm wavelength with an axial resolution of $5 \mu \mathrm{m}$ in tissue. The protocol of HD 5-Line Raster spaced at $0.25 \mathrm{~mm}$ was performed centering on the fovea, which is consisted of 6-mm parallel lines with 1024A-scans/B-scans and averaging 4 B-scans per image. There was no available enhanced-depth imaging (EDI) option on the version of the Cirrus HD-OCT used in the study. However, the single-line mode of the HD 5-line Raster scan manages 4096 consecutive axial scans along the same predetermined line. The CT was measured manually as the distance between the basal edge of the retinal pigment epithelium and the chorioscleral border using a caliper tool, at five points; directly beneath the fovea or the subfoveal area, and at the temporal and nasal points at a radius of 1500 and $3000 \mu \mathrm{m}$. The outer choroidal border can be well visualized on the single line HD 5 image in all patients included in the study (Figure 1). In total, three consecutive measurements were performed by one experienced ophthalmologist for all five points for each participant and the average of three measurements was used for statistical analyses.

Statistical analysis was performed using SPSS 21.0 for Windows (SPSS Inc., Chicago, IL, USA). The normality of the distribution of the study sample was assessed by the Shapiro-Wilk test. Independent samples $t$-test was used a

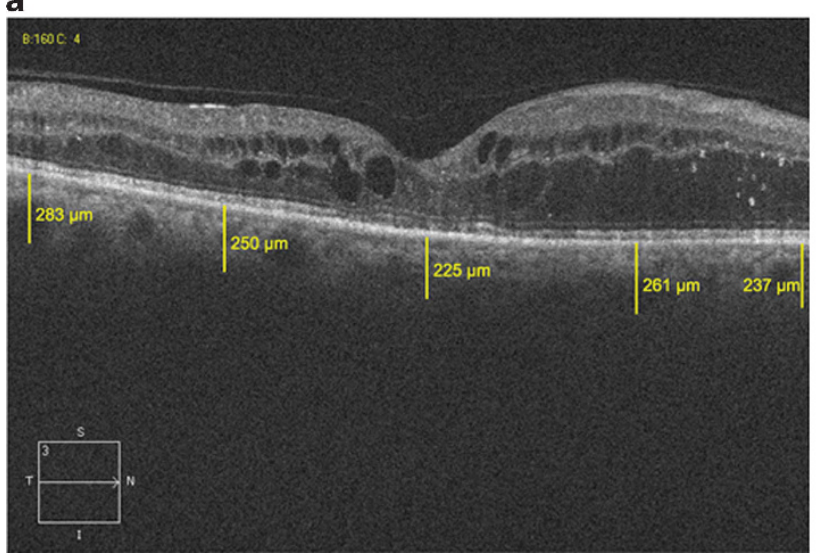

b

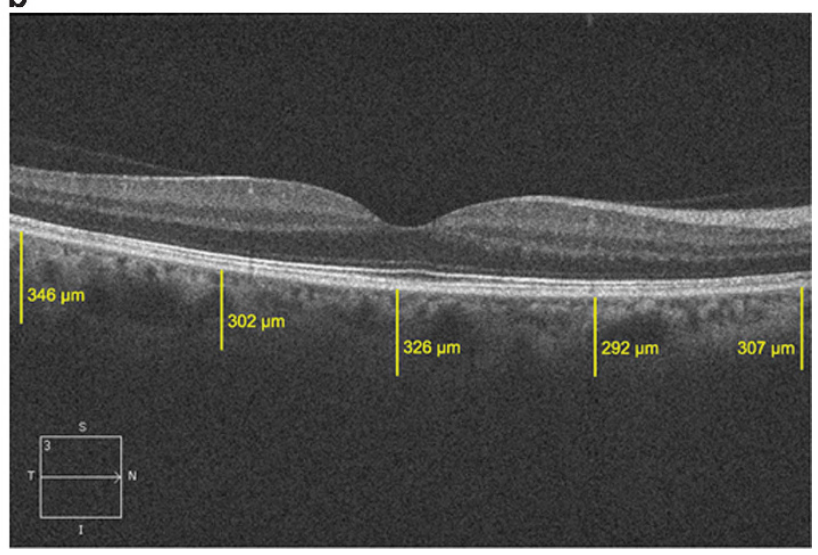

Figure 1 OCT image of a DME patient (a) and a healthy control (b) on Cirrus. White lines show choroidal thickness measurements retrieved perpendicularly from the outer edge of the hyper-reflective retinal pigment epithelium. It was measured at five points; the subfoveal area, and the temporal and nasal points at a radius of 1 and $3 \mathrm{~mm}$. 
Table 1 Comparison of demographics and baseline variables and outcomes in each group

\begin{tabular}{lccc}
\hline & DME (mean \pm SD) & Control (mean \pm SD) & P-value \\
\hline Age (years) & $61.03 \pm 7.13$ & $56.79 \pm 7.21$ & 0.019 \\
Gender (male/female) & $12 / 21$ & $13 / 21$ & 0.346 \\
Hemoglobin A1c (\%) & $7.3 \pm 1.7$ & $6.3 \pm 1.5$ & $<0.001$ \\
DME duration (months) & 16.2 & NA & NA \\
& & & NA \\
Lens Status (n) & 25 & 34 & $<0.001$ \\
$\quad$ Phakic & 9 & - & 0.36 \\
Pseudophakic & $0.72 \pm 0.57$ & 0.00 & 0.350 \\
BCVA (logMAR) & $0.42 \pm 0.79$ & $23.41 \pm 0.52$ & $<.75$ \\
SE (D) & $23.25 \pm 0.64$ & $212 \pm 20$ & $<.001$ \\
AL (mm) & $414 \pm 148$ & $17.19 \pm 2.14$ & $<.091$ \\
CFT ( $\mu$ m) & $18.41 \pm 3.50$ & $3.52 \pm 1.03$ & $<0.001$ \\
IOP (mm Hg) & $2.58 \pm 0.96$ & & \\
OPA & & & \\
\hline A & & & \\
\hline
\end{tabular}

Abbreviations: AL, axial length; BCVA, best-corrected visual acuity; CFT, central foveal thickness; D, diopters; DME, diabetic macular edema; IOP, intraocular pressure; $n$, number; NA, not applicable; OPA, ocular pulse amplitude; SE, spherical equivalent.

$P<0.05$ indicates statistically significance.

to analyze the differences in the measured parameters between controls and DME patient. A linear regression analysis was used to evaluate the effect of the OPA on the CT measurements. Pearson's correlation coefficient was used to evaluate the correlation between IOP and OPA measurements. $P$-value $<0.05$ were considered to be statistically significant.

\section{Results}

\section{Baseline characteristics}

Complete baseline characteristics and outcomes are listed in Table 1. The mean age of DME patients and control subjects was $61.0 \pm 7.1$ and $56.7 \pm 7.2$ years, respectively $(P=0.019)$. In the DME group 25 eyes were phakic $(73 \%)$ and $34(100 \%)$ were phakic in the control subjects. The mean $\mathrm{HbA} 1 \mathrm{c}$ were $7.3 \pm 1.7$ and $6.3 \pm 1.5$ in DME and controls, respectively $(P<0.001)$. The mean BCVA of DME patients and controls were significantly different $(P<0.001$; Table 1). The mean SE was not significantly different between groups $(P=0.36)$. The mean $\mathrm{AL}$ of DME patients and controls were $23.25 \pm 0.64 \mathrm{~mm}$ and $23.41 \pm 0.75 \mathrm{~mm}$, respectively, and the difference was not statistically significant $(P=0.350)$. The mean central foveal thickness was $414 \pm 148 \mu \mathrm{m}$ in DME and $212 \pm 20 \mu \mathrm{m}$ in control subjects $(P<0.001)$. The mean IOP values were $18.4 \pm 3.5$ and $17.1 \pm 2.1 \mathrm{~mm} \mathrm{Hg}$ in DME patients and healthy controls, respectively. IOP values did not show significant difference between the groups $(P=0.091)$. The mean OPA values in patients with DME $(2.58 \pm 0.96)$ and controls $(3.52 \pm 1.03)$ were statistically different $(P<0.001)$.

The IOP showed a significantly positive correlation with OPA in both DME $(P=0.002, r=0.526)$ and controls $(P=0.004, r=0.483)$.
Table 2 The mean choroidal thickness measurements of DME patients and healthy controls

\begin{tabular}{lcrc}
\hline Choroidal thickness $(\mu \mathrm{m})$ & $\begin{array}{c}\text { DME } \\
(\text { mean } \pm S D)\end{array}$ & $\begin{array}{c}\text { Control } \\
(\text { mean } \pm S D)\end{array}$ & P-value \\
\hline Subfoveal & $273.5 \pm 30.2$ & $321.4 \pm 36.5$ & $<0.001$ \\
Nasal, $1.5 \mathrm{~mm}$ & $273.2 \pm 29.4$ & $325.0 \pm 41.4$ & $<0.001$ \\
Temporal, $1.5 \mathrm{~mm}$ & $279.9 \pm 27.6$ & $319.5 \pm 41.0$ & $<0.001$ \\
Nasal, $3 \mathrm{~mm}$ & $275.3 \pm 27.1$ & $327.6 \pm 36.8$ & $<0.001$ \\
Temporal, 3 mm & $277.5 \pm 28.4$ & $322.4 \pm 40.7$ & $<0.001$ \\
\hline
\end{tabular}

$P<0.05$ indicates statistically significance.

\section{CT measurements}

The mean subfoveal CT value was $273.5 \pm 30.2 \mu \mathrm{m}$ in the eyes with DME and $321.4 \pm 36.5 \mu \mathrm{m}$ in the control group. The difference was statistically significant $(P<0.001)$. Similarly, statistically significant difference was found for nasal and temporal CT measurements between the two groups $(P<0.001$; Table 2$)$. Linear regression analysis did not reveal any significant association between OPA and CT measurements in DME patients ( $P$-values ranged between 0.298 and 0.943$)$ and controls ( $P$-values ranged between 0.262 and 0.319; Supplementary Table 1).

According to linear regression analysis age had no effect on the CT measurements in DME patients ( $P$ values ranged between 0.649 and 0.945$)$ and controls $(P$ values ranged between 0.702 and 0.947 ).

\section{Discussion}

To our knowledge, this is the first study investigating the relationship between CT and OPA in treatment-naive patients with newly diagnosed DME. The results demonstrated that the CT and OPA were significantly 
decreased in DME patients; however, they did not show any significant correlation.

The function of the choroid is to transport blood and nutrients to the outer retinal layers. ${ }^{14}$ The choroidal thinning observed by serial EDI OCT imaging is corroborated by histopathologic findings of narrowing of choroidal arterioles, choriocapillaris atrophy, and capillary dropout in eyes with diabetic retinopathy. ${ }^{15,16}$ Langham et $\mathrm{al}^{17}$ performed an ocular hemodynamic study revealing a progressive decrease in choroidal blood flow with increasing severity of the underlying diabetic retinopathy. Similarly, laser Doppler flowmetry studies have shown reduced choroidal vascular flow and volume in eyes with diabetic retinopathy. ${ }^{18}$ The decrease in observed blood flow may be attributable to an increase in vascular resistance from capillary dropout and choriocapillaris atrophy. ${ }^{2}$ These changes may result in ischemia to the outer retina and RPE, which leads to an increase in VEGF expression in the RPE and microvascular endothelial cells, potentiating breakdown of the blood-retinal barrier, and ultimately resulting in DME.${ }^{19}$ Hence, the choroid may indeed have a significant role in the pathophysiology of DME.

$\mathrm{CT}$ studies in diabetes have produced diverging results, however, with some reports suggesting choroidal thickening, thinning, and no change in eyes with diabetic retinopathy. CT measurements in eyes with DME similarly have been inconsistent. ${ }^{2,20}$ One important explanation for these variable results is the significant variability of $\mathrm{CT}$ in these retrospective, cross-sectional studies. CT has been shown to vary with age, ${ }^{21}$ refractive error, ${ }^{22}$ and even time of day. ${ }^{23}$ To overcome the effect of these variables we compared the CT measurements in sex and spherical refraction matched DME and controls that were performed at the same time of the day to avoid diurnal variations. However, the age was statistically different between study groups. To overcome this limitation we used a regression model and found no significant correlation between CT measurements and age for both groups.

Moreover, many of these studies include eyes that received treatment with intravitreal anti-VEGF therapy, which has been shown to cause choroidal thinning in other diseases such as age-related macular degeneration. ${ }^{24,25}$ Studies have demonstrated that VEGF secreted by RPE cells has a trophic role on the choroidal vasculature. ${ }^{26}$ Therefore, blocking the action of VEGF on the choroid with anti-VEGF therapy, the permeability of the choroidal vasculature decreases, which can be noted by a decrease in CT. Lains $e t \mathrm{al}^{3}$ demonstrated a significant decrease in $\mathrm{CT}$ in DME patients following treatment with anti-VEGF therapy. In a more recent study by Yiu et al, ${ }^{27} \mathrm{CT}$ was also found to decrease significantly in patients receiving anti-VEGF therapy. In this study we included patients without prior anti-VEGF treatment to overcome this limitation. By choosing to include only treatment-naive patients our study is better able to examine the CT in DME.

OPA is caused by cardiovascular pulsations and ocular blood flow and is a hemodynamic parameter reflecting mainly choroidal perfusion. ${ }^{9,10}$ The OPA has been used in many studies as an indirect measure of the choroidal perfusion. Differences in IOP values during systole and diastole are used to calculate an index that estimates ocular blood supply, of which $70-80 \%$ is choroidal. ${ }^{28}$ There are many different methods for measuring the OPA and choroidal perfusion. ${ }^{29}$ Pascal DCT is designed to measure IOP and OPA, and hence it can also provide choroidal hemodynamics global data, mainly on the pulsatile choroidal component. DCT is more accurate than Langham ocular blood flow ${ }^{30}$ in measuring the IOP and OPA and was recently shown to have good concordance with intracameral IOP. ${ }^{31}$ Recent studies showed a positive correlation between DCTs OPA and IOP. ${ }^{32}$ Similar to the reports in literature they were positively correlated in our study population.

OPA values were significantly reduced in our DME patients compared with the age and sex-matched healthy subjects. However, OPA did not show any significant correlation with the CT measurements in both groups. Similar results were also reported by the recent novel studies on chronic heavy smokers ${ }^{33}$ and acute migraine attack $^{34}$ confirming decreased OPA values along with decreased CT. The OPA is mainly the result of global choroidal pulsation; however, the subfoveal $\mathrm{CT}$ is measured only at the posterior pole. The currently available OCT devices can measure the $\mathrm{CT}$ in this limited area. Therefore, we do not actually know whether these parameters are correlated unless the mean global CT is measured. Furthermore, as OPA is mainly the result of global choroidal pulsation; it may not be the best method to evaluate the possibility of choroidal perfusion abnormalities in the macular area. To assess these choroidal circulation abnormalities, other methods including semiquantitative indocyanine green angiography can be used. Our results may also be associated with the variability of participants included in the study.

This study has some limitations that should be considered. Although the HD 5-line Raster images have very high resolution, the choroid-sclera junction could not be visualized with absolute certainty in some patients. However, we did not include those subjects because we were not able to mark the choroidal outer boundary with certainty.

In addition, the power of the current study was fairly limited. To overcome this limitation, we have 
performed a linear regression analysis in this normally distributed sample.

In conclusion, the current study suggests that both pulsatile choroidal blood flow and CT are decreased in patients with DME without prior anti-VEGF treatment. However, no correlation was found between these parameters in DME. Further studies including histopathological changes should be performed to reveal the choroidal tissue changes in DME, as CT measurements cannot show the ultrastructural changes.

\section{Summary}

\section{What was known before}

- Choroidal thickness (CT) is decreased in patients with diabetic macular edema (DME).

\section{What this study adds}

- Both pulsatile choroidal blood flow (ocular pulse amplitude) and CT are decreased in patients with DME without prior anti-vascular endothelial growth factor treatment.

\section{Conflict of interest}

The authors declare no conflict of interest.

\section{References}

1 Romero-Aroca P. Managing diabetic macular edema: the leading cause of diabetes blindness. World J Diabetes 2011; 2(6): 98-104.

2 Querques G, Lattanzio R, Querques L, Del Turco C, Forte R, Pierro L et al. Enhanced depth imaging optical coherence tomography in type 2 diabetes. Invest Ophthalmol Vis Sci 2012; 53(10): 6017-6024.

3 Lains I, Figueira J, Santos AR, Baltar A, Costa M, Nunes S et al. Choroidal thickness in diabetic retinopathy: the influence of antiangiogenic therapy. Retina 2014; 34(6): 1199-1207.

4 Adhi M, Brewer E, Waheed NK, Duker JS. Analysis of morphological features and vascular layers of choroid in diabetic retinopathy using spectral-domain optical coherence tomography. JAMA Ophthalmol 2013; 131(10): 1267-1274.

5 Regatieri CV, Branchini L, Carmody J, Fujimoto JG, Duker JS. Choroidal thickness in patients with diabetic retinopathy analyzed by spectral-domain optical coherence tomography. Retina 2012; 32(3): 563-568.

6 Rayess N, Rahimy E, Ying GS, Bagheri N, Ho AC, Regillo CD et al. Baseline choroidal thickness as a predictor for response to anti-vascular endothelial growth factor therapy in diabetic macular edema. Am J Ophthalmol 2014; 159: 85-91 e1-3.

7 Gerendas BS, Waldstein SM, Simader C, Deak G, Hajnajeeb B, Zhang L et al. Three-dimensional automated choroidal volume assessment on standard spectral-domain optical coherence tomography and correlation with the level of diabetic macular edema. Am J Ophthalmol 2014; 158: 1039-1048.
8 Rayess N, Rahimy E, Ying GS, Bagheri N, Ho AC, Regillo CD et al. Baseline choroidal thickness as a predictor for response to anti-vascular endothelial growth factor therapy in diabetic macular edema. Am J Ophthalmol 2015; 159(1): 85-91 e83.

9 Walker RE, Compton GA, Langham ME. Pneumatic applanation tonometex studies. IV. Analysis of pulsatile response. Exp Eye Res 1975; 20(3): 245-253.

10 Walker RE, Langham ME. Pneumatic applanation tonometer studies. III. Analysis of the floating tip sensor. Exp Eye Res 1975; 20(2): 167-172.

11 Photocoagulation for diabetic macular edema. Early Treatment Diabetic Retinopathy Study report number 1. Early Treatment Diabetic Retinopathy Study research group. Arch Ophthalmol 1985; 103(12): 1796-1806.

12 Brown DM, Nguyen QD, Marcus DM, Boyer DS, Patel S, Feiner L et al. Long- term outcomes of ranibizumab therapy for diabetic macular edema: the 36- month results from two phase III trials: RISE and RIDE. Ophthalmology 2013; 120(10): 2013-2022.

13 Lee SW, Yu SY, Seo KH, Kim ES, Kwak HW. Diurnal variation in choroidal thickness in relation to sex, axial length, and baseline choroidal thickness in healthy Korean subjects. Retina 2014; 34(2): 385-393.

14 Nickla DL, Wallman J. The multifunctional choroid. Prog Retin Eye Res 2010; 29(2): 144-168.

15 Hidayat AA, Fine BS. Diabetic choroidopathy. Light and electron microscopic observations of seven cases. Ophthalmology 1985; 92(4): 512-522.

16 Lutty GA, Cao J, McLeod DS. Relationship of polymorphonuclear leukocytes to capillary dropout in the human diabetic choroid. Am J Pathol 1997; 151(3): 707-714.

17 Langham ME, Grebe R, Hopkins S, Marcus S, Sebag M. Choroidal blood flow in diabetic retinopathy. Exp Eye Res 1991; 52(2): 167-173.

18 Schocket LS, Brucker AJ, Niknam RM, Grunwald JE, DuPont J, Brucker AJ. Foveolar choroidal hemodynamics in proliferative diabetic retinopathy. Int Ophthalmol 2004; 25(2): 89-94.

19 Mori F, Hikichi T, Takahashi J, Nagaoka T, Yoshida A. Dysfunction of active transport of blood-retinal barrier in patients with clinically significant macular edema in type 2 diabetes. Diabetes Care 2002; 25(7): 1248-1249.

20 Kim JT, Lee DH, Joe SG, Kim JG, Yoon YH. Changes in choroidal thickness in relation to the severity of retinopathy and macular edema in type 2 diabetic patients. Invest Ophthalmol Vis Sci 2013; 54(5): 3378-3384.

21 Margolis R, Spaide RF. A pilot study of enhanced depth imaging optical coherence tomography of the choroid in normal eyes. Am J Ophthalmol 2009; 147(5): 811-815.

22 Fujiwara T, Imamura Y, Margolis R, Slakter JS, Spaide RF. Enhanced depth imaging optical coherence tomography of the choroid in highly myopic eyes. Am J Ophthalmol 2009; 148 (3): 445-450.

23 Tan CS, Ouyang Y, Ruiz H, Sadda SR. Diurnal variation of choroidal thickness in normal, healthy subjects measured by spectral domain optical coherence tomography. Invest Ophthalmol Vis Sci 2012; 53(1): 261-266.

24 Branchini L, Regatieri C, Adhi M, Flores-Moreno I, Manjunath V, Fujimoto JG et al. Effect of intravitreous antivascular endothelial growth factor therapy on choroidal thickness in neovascular age-related macular degeneration using spectral-domain optical coherence tomography. JAMA Ophthalmol 2013; 131(5): 693-694. 
25 Yamazaki T, Koizumi H, Yamagishi T, Kinoshita S. Subfoveal choroidal thickness after ranibizumab therapy for neovascular age-related macular degeneration: 12-month results. Ophthalmology 2012; 119(8): 1621-1627.

26 Maharaj AS, D'Amore PA. Roles for VEGF in the adult. Microvasc Res 2007; 74(2-3): 100-113.

27 Yiu G, Manjunath V, Chiu SJ, Farsiu S, Mahmoud TH. Effect of anti-vascular endothelial growth factor therapy on choroidal thickness in diabetic macular edema. Am J Ophthalmol 2014; 158(4): 745-751 e742.

28 Kaufmann C, Bachmann LM, Thiel MA. Intraocular pressure measurements using dynamic contour tonometry after laser in situ keratomileusis. Invest Ophthalmol Vis Sci 2003; 44(9): 3790-3794.

29 Schmetterer L, Dallinger S, Findl O, Strenn K, Graselli U, Eichler HG et al. Noninvasive investigations of the normal ocular circulation in humans. Invest Ophthalmol Vis Sci 1998; 39(7): 1210-1220.
30 Langham ME FR, O'Brien V et al. (eds) Noninvasive Measurement of Pulsatile Blood Flow in the Human Eye. Kugler and Ghedini: Amsterdam, The Netherlands, 1989.

31 Boehm AG, Weber A, Pillunat LE, Koch R, Spoerl E. Dynamic contour tonometry in comparison to intracameral IOP measurements. Invest Ophthalmol Vis Sci 2008; 49(6): 2472-2477.

32 Kaufmann C, Bachmann LM, Thiel MA. Comparison of dynamic contour tonometry with goldmann applanation tonometry. Invest Ophthalmol Vis Sci 2004; 45(9): 3118-3121.

33 Dervişoğulları MS, Totan Y, Tenlik A, Yuce A. Effects of cigarette smoking on choroidal and retinal thickness and ocular pulse amplitude. Cutan Ocul Toxicol 2015; 34(3): 217-221.

34 Dervisogullari MS, Totan Y, Gencler OS. Choroid thickness and ocular pulse amplitude in migraine during attack. Eye (Lond) 2015; 29: 371-375.

Supplementary Information accompanies this paper on Eye website (http://www.nature.com/eye) 